\title{
On the Approximation of the Generalized-K Distribution by a Gamma Distribution for Modeling Composite Fading Channels
}

\author{
Saad Al-Ahmadi, Member, IEEE, and Halim Yanikomeroglu, Member, IEEE
}

\begin{abstract}
In wireless channels, multipath fading and shadowing occur simultaneously leading to the phenomenon referred to as composite fading. The use of the Nakagami probability density function (PDF) to model multipath fading and the Gamma PDF to model shadowing has led to the generalized- $K$ model for composite fading. However, further derivations using the generalized- $K$ PDF are quite involved due to the computational and analytical difficulties associated with the arising special functions. In this paper, the approximation of the generalized$K$ PDF by a Gamma PDF using the moment matching method is explored. Subsequently, an adjustable form of the expressions obtained by matching the first two positive moments, to overcome the arising numerical and/or analytical limitations of higher order moment matching, is proposed. The optimal values of the adjustment factor for different integer and non-integer values of the multipath fading and shadowing parameters are given. Moreover, the approach introduced in this paper can be used to well-approximate the distribution of the sum of independent generalized- $K$ random variables by a Gamma distribution; the need for such results arises in various emerging distributed communication technologies and systems such as coordinated multipoint transmission and reception schemes including distributed antenna systems and cooperative relay networks.
\end{abstract}

Index Terms-Composite fading, Gamma distribution, generalized- $K$ distribution, moment matching, positive and negative moments, lower and upper tails, network MIMO, distributed antenna systems, radar and sonar.

\section{INTRODUCTION}

$\mathbf{M}$ ODELING composite fading channels is essential for the analysis of several wireless communication problems including interference analysis in cellular systems and performance analysis of network MIMO, distributed antenna systems, and cooperative relay networks. The small-scale multipath fading is often modeled using Rayleigh, Rician, or Nakagami distribution. The latter one is versatile enough to encompass the Rayleigh distribution as a special case and to approximate the Rician distribution. The large-scale fading (shadowing) is often modeled using a lognormal distribution (refer to [1] and the references therein). However, the lognormal-based composite fading models $[2,3]$ do not lead to closed-form expressions for the received signal power distribution which hampers further analytical derivations. As an

Manuscript received September 20, 2008; revised March 19, 2009 and May 28, 2009; accepted July 27, 2009. The associate editor coordinating the review of this paper and approving it for publication was S. Gassemzadeh.

The authors are with the Department of Systems and Computer Engineering, Carleton University, Ottawa, Canada (e-mail: \{saahmadi, halim\}@sce.carleton.ca).

S. Al-Ahmadi's work was supported by King Fahd University of Petroleum and Minerals, Saudi Arabia.

Digital Object Identifier 10.1109/TWC.2010.02.081266 alternative, it has been proposed to use the Gamma distribution to model large-scale fading where it has been observed that the Gamma distribution fits the experimental data, and it closely approximates the lognormal distribution [4-7].

The use of the Gamma distribution to model shadowing and the Nakagami distribution to model the small-scale random variations of the received signal envelope, has led to a closedform expression of the composite fading probability density function (PDF) known as the Gamma-Gamma (generalized$K)$ PDF. The Gamma-Gamma model was introduced to model scattering in radar [8] and reverberation in sonar [9] and has recently generated interest in wireless communications as well [10-13]. However, further derivations using that model have shown to be analytically difficult or computationally involved due to the arising special functions.

In this paper, the approximation of the generalized- $K$ distribution by a Gamma distribution through matching both positive and negative moments is explored. The obtained results have shown that matching the higher order moments leads to a good approximation, up to a certain level of accuracy, in both the upper and lower tail regions, and may lead to lower and upper bounds on the approximated cumulative distribution function (CDF). However, such a matching has two main limitations: (i) it results in involved expressions that are difficult to handle and complicated to draw insights from; (ii) negative moments may not exist for small values of the multipath fading and shadowing parameters. Subsequently, an adjusted form of the expressions obtained by matching the first two positive moments is introduced to closely approximate the generalized- $K$ composite fading PDF by the simple and tractable Gamma PDF. This region-wise approximation yields sufficient accuracy for a broad range of integer and non-integer values of the multipath fading and shadowing parameters. Moreover, the introduced method can be used to approximate the PDF of the sum of independent generalized- $K$ random variables (RVs) in the lower and upper tail regions. Finally, since the approximating Gamma model allows the use of the closed-form expressions developed in literature for Nakagami fading channels [14-15], some performance analysis applications, out of many, are stated.

\section{The Composite Fading Model And Related WORK}

When the random variation of the envelope of the received signal, due to small-scale multipath fading, is modeled by the Nakagami distribution [16], the PDF of the received power $\gamma$, 
conditioned on the average local power $\Omega$, takes the form of a Gamma distribution:

$$
\begin{aligned}
& p_{\gamma / \Omega}(x)= \\
& \frac{1}{\Gamma\left(m_{m}\right)}\left(\frac{m_{m}}{\Omega}\right)^{m_{m}} x^{m_{m}-1} \exp \left(-\frac{m_{m} x}{\Omega}\right), x>0, m_{m} \geq 0.5,
\end{aligned}
$$

where $\Gamma(\cdot)$ is the Gamma function and $m_{m}$ is the Nakagami multipath fading parameter.

The variation of the average local power, due to shadowing, is usually modeled by the lognormal distribution [3]. However, the analytically better tractable Gamma distribution has also shown a good fit to data obtained through propagation measurements $[5,6]$, besides it can approximate the lognormal distribution for the relevant range of shadowing severity in wireless channels $[5,7]$ :

$$
\begin{aligned}
& p_{\Omega}(y)= \\
& \frac{1}{\Gamma\left(m_{s}\right)}\left(\frac{m_{s}}{\Omega_{0}}\right)^{m_{s}} y^{m_{s}-1} \exp \left(-\frac{m_{s}}{\Omega_{0}} y\right), y>0, m_{s}>0 .
\end{aligned}
$$

In (2), $m_{s}$ is the shadowing parameter and $\Omega_{0}$ is the mean of the received local power. Similar to the multipath parameter $m_{m}$, the severity of shadowing is inversely proportional to $m_{s}$ so that small values of $m_{s}$ indicate severe shadowing conditions. In [5, 7], using the moment matching method between the Gamma PDF in (2) and the lognormal PDF, it was shown that $m_{s}=\frac{1}{e^{\left(\sigma_{s} / 8.686\right)^{2}-1}}$ where $\sigma_{s}$ denotes the standard deviation in the lognormal shadowing model.

Using (1) and (2), the PDF of $\gamma$ can be derived as $[8-10]^{1}$

$$
\begin{aligned}
p_{\gamma}(x)= & \frac{2}{\Gamma\left(m_{s}\right) \Gamma\left(m_{m}\right)} b^{m_{s}+m_{m}} x^{\left(\frac{m_{s}+m_{m}}{2}\right)-1} \\
& \times K_{m_{s}-m_{m}}(2 b \sqrt{x}), x>0, m_{m} \geq 0.5, m_{s}>0,
\end{aligned}
$$

where $K_{m_{s}-m_{m}}(\cdot)$ is the modified Bessel function of the second kind and order $\left(m_{s}-m_{m}\right)$ and $b=\sqrt{\frac{m_{m} m_{s}}{\Omega_{0}}}$. The PDF in (3) appeared first in [8] where the instantaneous power is assumed to follow a Gamma distribution whose mean is also assumed to have a Gamma distribution. The PDF in (3) is known as the generalized- $K$ model $^{2}$ and the McDaniel model in wireless and sonar literature, respectively ([10] and references therein).

The CDF of $\gamma$, was derived in [13] as

$$
\begin{aligned}
P(\gamma)=\pi \csc (\pi \alpha) & {\left[\frac{\left(b^{2} \gamma\right)^{m_{m}}{ }_{1} F_{2}\left(m_{m} ; 1-\alpha, 1+m_{m} ; b^{2} \gamma\right)}{\Gamma\left(m_{s}\right) \Gamma(1-\alpha) \Gamma\left(m_{m}+1\right)}\right.} \\
- & \left.\frac{\left(b^{2} \gamma\right)^{m_{s}}{ }_{1} F_{2}\left(m_{s} ; 1+\alpha, 1+m_{s} ; b^{2} \gamma\right)}{\Gamma\left(m_{m}\right) \Gamma(1+\alpha) \Gamma\left(m_{s}+1\right)}\right],
\end{aligned}
$$

where $\alpha=m_{s}-m_{m}$ and ${ }_{p} F_{q}$ is the generalized hypergeometric function for integer $p$ and $q$ [20].

However, as pointed out in [9], the computation of such a $\mathrm{CDF}$ expression which contains the hyper-geometric function

\footnotetext{
${ }^{1}$ In fact, the distribution of the product of $M$ independent Gamma RVs, where the generalized- $K$ PDF corresponds to the case where $M=2$, was derived in [17]. Moreover, the generalized- $K$ distribution belongs to the (Fox) $H$-function distributions family [18].

${ }^{2}$ It should be highlighted here that in literature, the notion "generalized- $K$ " was used to denote another similar distribution [19].
}

term is not straightforward due to the associated numerical instabilities that will require the use of approximations and asymptotic expansions or the numerical inversion of the characteristic function. Moreover, further derivations using the characteristic function approach, such as the PDF of the sum of $N$ generalized- $K \mathrm{RVs}$, are quite involved even for the independent and identically distributed (i.i.d.) case due to the difficulties associated with the Whittaker function [21].

\section{Approximation Using the Moment Matching METHOD}

An alternative approach, to avoid the analytical difficulties, is to consider approximating the PDF in (3) by a more tractable PDF using the moment matching method. We propose using the Gamma distribution due to the following reasons: (i) Gamma distribution is a Type-III Pearson distribution which is widely used in fitting distributions for positive RVs by matching the first and second moments [18], and (ii) since the PDF in (3) corresponds to the product of two Gamma $\mathrm{RVs}$, one of the corresponding Gamma PDFs will dominate for large values of $m_{m}$ or $m_{s}$ [7].

The $n^{\text {th }}$ moment of the generalized- $K$ distribution can be derived as [13]

$$
E\left[\gamma^{n}\right]=\mu_{n}=\frac{\Gamma\left(m_{m}+n\right) \Gamma\left(m_{s}+n\right)}{\Gamma\left(m_{m}\right) \Gamma\left(m_{s}\right)}\left(\frac{\Omega_{0}}{m_{m} m_{s}}\right)^{n},
$$

where $E[\cdot]$ denotes the statistical expectation.

Denoting $Z \sim \mathcal{G}(k, \theta)$ as a Gamma distributed RV with a shape parameter $k$ and a scale parameter $\theta$, the PDF of $Z$ is given as [22]

$$
p_{Z}(x)=\frac{\theta^{-k}}{\Gamma(k)} x^{k-1} \exp (-x / \theta), \quad x>0 .
$$

Furthermore, the $n^{\text {th }}$ moment of the Gamma distribution can be expressed as [22]

$$
E\left[z^{n}\right]=\mu_{n}^{(\mathcal{G})}=\frac{\Gamma(k+n) \theta^{n}}{\Gamma(k)} .
$$

Now, using the expressions in (5) and (7), the first, second, and third moments of the generalized- $K$ distribution and the approximating Gamma distribution can be matched as

$$
\begin{aligned}
k \theta & =\Omega_{0}, \\
\theta^{2} k(k+1) & =K_{1} \Omega_{0}^{2}, \\
\theta^{3} k(k+1)(k+2) & =K_{2} K_{1} \Omega_{0}^{3} .
\end{aligned}
$$

On the other hand, the negative moments, as defined in [23], of the generalized- $K$ PDF and the Gamma PDF can be expressed using the expressions in (5) and (7) as

$$
\begin{gathered}
\theta(k-1)=K_{-1} \Omega_{0}, k>1, m_{m}>1, m_{s}>1, \\
\theta^{2}(k-2)(k-1)=K_{-1} K_{-2} \Omega_{0}^{2}, k>2, m_{m}>2, m_{s}>2,
\end{gathered}
$$


where

$$
\begin{aligned}
K_{1} & =\frac{\left(m_{m}+1\right)\left(m_{s}+1\right)}{m_{m} m_{s}}=1+\frac{1}{m_{m}}+\frac{1}{m_{s}}+\frac{1}{m_{m} m_{s}} \\
K_{2} & =\frac{\left(m_{m}+2\right)\left(m_{s}+2\right)}{m_{m} m_{s}}=1+\frac{2}{m_{m}}+\frac{2}{m_{s}}+\frac{4}{m_{m} m_{s}} \\
K_{-1} & =\frac{\left(m_{m}-1\right)\left(m_{s}-1\right)}{m_{m} m_{s}}=1-\frac{1}{m_{m}}-\frac{1}{m_{s}}+\frac{1}{m_{m} m_{s}} \\
K_{-2} & =\frac{\left(m_{m}-2\right)\left(m_{s}-2\right)}{m_{m} m_{s}}=1-\frac{2}{m_{m}}-\frac{2}{m_{s}}+\frac{4}{m_{m} m_{s}}
\end{aligned}
$$

Matching different pairs of moments will result in the scale and shape parameters for the approximating Gamma PDF as shown in Table I. In Table I, $\theta_{i, j}$ and $k_{i, j}$ denote the scale and shape parameters of the approximating Gamma PDF obtained by matching the $i^{\text {th }}$ and the $j^{\text {th }}$ moments, respectively.

Now, examining the expressions of the approximating Gamma PDF parameters given in Table I, the following may be stated:

- The scale parameter of the approximating Gamma PDF obtained by matching the positive moments is larger than the one obtained by matching the negative moments. For example, it can be easily seen that $\theta_{1,2}=\theta_{1,-1}+$ $\frac{2}{m_{m} m_{s}} \Omega_{0}$. Since the negative moments characterize a distribution at the origin [23] (the lower tail for a positive $\mathrm{RV}$ ) and the positive moments characterize a distribution at the upper tail, we may conclude that the generalizedK PDF (CDF) can be approximated by a Gamma distribution whose scale and shape parameters depend on the region of the PDF (CDF) of interest. Such a regionwise (piece-wise) approximation was used in [24] to well-approximate the sum of lognormal RVs by a single lognormal RV.

- Matching moments for $n \geq 2$ will lead to involved expressions as seen in Table I. Moreover, not including the first positive moment in the moments matched results in an approximating Gamma PDF that does not have the same mean as the approximated generalized- $K$ PDF (i.e., the generalized- $K$ PDF and the approximating Gamma PDF have different average power values).

- Matching negative moments may not be possible for small values of $m_{m}$ and/or $m_{s}$ as indicated in (11), (12) and subsequent expressions in Table I.

- The scale and shape parameters of the approximating Gamma distribution are dependent on the fading parameters in the sense that as $m_{m}$ and/or $m_{s}$ increase, the difference between the predicted scale parameters decreases and hence the difference between the approximating PDFs (CDFs) becomes small. So, for small values of $m_{m}$ and/or $m_{s}$ (while $m_{m}, m_{s}>2$ ), the difference between the two approximating Gamma CDFs might be large enough to bound the approximated CDF in the lower tail region as seen in Fig. 1. On the other hand, matching the lower order moments for large values of $m_{m}$ and/or $m_{s}$ does not result in a good approximation as seen in Figs. 2 and 3 since the approximating CDFs are too close to each other.

Note: In Figs. 1-3, the complementary cumulative distribution function (CCDF), and particularly the region corresponding to $P(X \geq x) \leq 0.1$, is shown for the upper tail region to obtain more illustrative results.
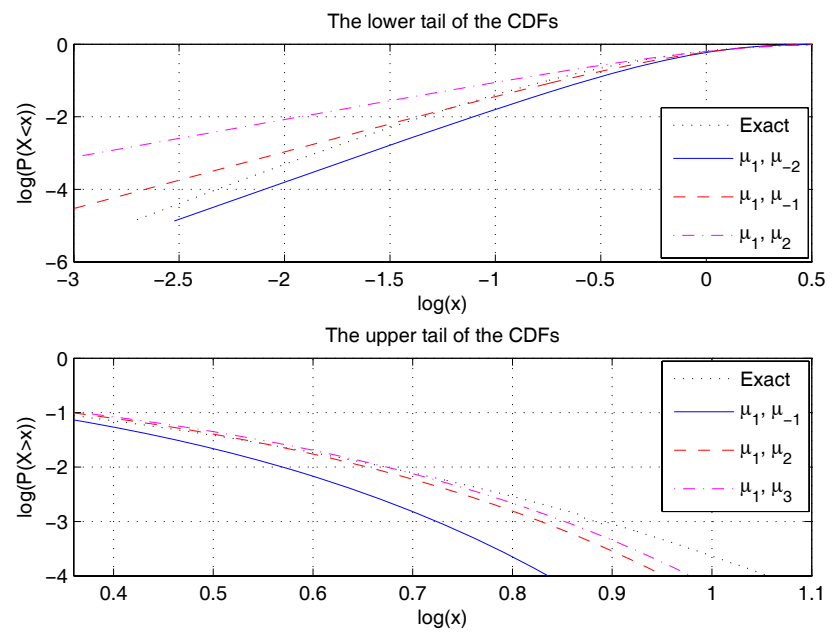

Fig. 1. The log-log CDF plots of the generalized- $K$ and the approximating Gamma RVs for $m_{m}=2.5$ and $m_{s}=2.5$ using the moment matching method.
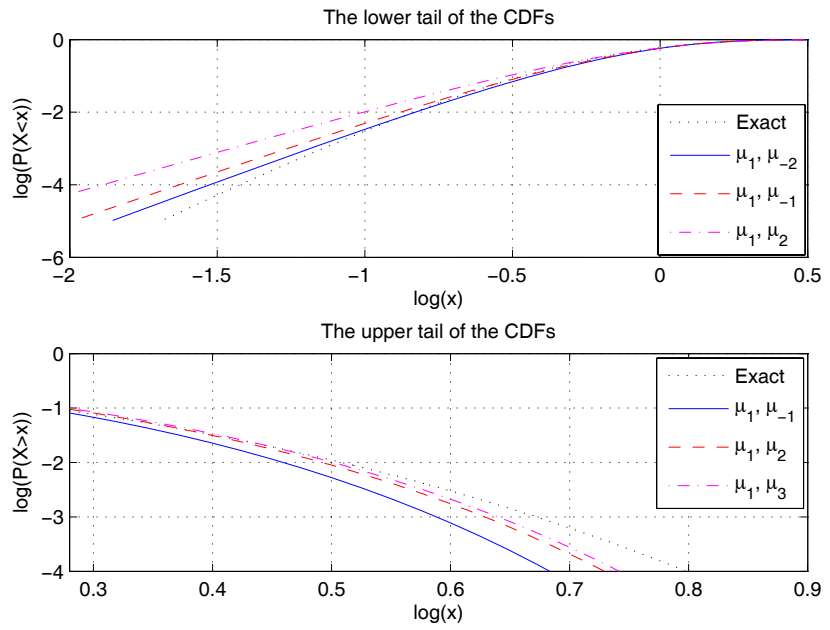

Fig. 2. The log-log CDF plots of the generalized- $K$ and the approximating Gamma RVs for $m_{m}=7$ and $m_{s}=4$ using the moment matching method.

\section{The Moment Matching Method with ADJUSTMENT}

In order to bypass the limitations explained before on the use of the moment matching for higher order moments, we may consider an adjustable form for the scale and shape parameters of the approximating Gamma PDF obtained by matching only the first two positive moments since (i) these expressions, as given in Table I, are simple and valid for all values of $m_{m}$ and $m_{s}$, and (ii) the first positive moment is included in the matching.

First, we may re-write the scale and shape parameters using Table I as

$$
\begin{aligned}
\theta_{1,2} & =\left[\frac{1}{m_{m}}+\frac{1}{m_{s}}+\frac{1}{m_{m} m_{s}}\right] \Omega_{0} \\
& =[\mathrm{AF}] \Omega_{0}, 0 \leq \mathrm{AF} \leq \mathrm{AF}_{\max } \\
k_{1,2} & =\frac{1}{\mathrm{AF}}, 0 \leq \mathrm{AF} \leq \mathrm{AF}_{\max }
\end{aligned}
$$

In the above, the term $\frac{1}{m_{m}}+\frac{1}{m_{s}}+\frac{1}{m_{m} m_{s}}$ is the amount of fading (AF) in the composite fading channel as derived 
TABLE I

EXPRESSIONS OF THE SCALE AND THE SHAPE PARAMETERS OF THE APPROXIMATING GAMMA PDF OBTAINED BY MOMENT MATCHING (FOR $K_{1}, K_{2}$, $K_{-1}, K_{-2}$, REFER TO (13A)-(13D))

\begin{tabular}{|l|c|c|}
\hline Matched moments & Scale parameter & Shape parameter \\
\hline$\mu_{1}, \mu_{2}$ & $\theta_{1,2}=\left(K_{1}-1\right) \Omega_{0}, \theta_{1,2}>0$ & $k_{1,2}=\frac{1}{K_{1}-1}, k_{1,2}>0$ \\
\hline$\mu_{1}, \mu_{3}$ & $\theta_{1,3}=\frac{\left(-3+\sqrt{9+8\left(K_{1} K_{2}-1\right)}\right) \Omega_{0}}{4}, \theta_{1,3}>0$ & $k_{1,3}=\frac{4}{-3+\sqrt{9+8\left(K_{1} K_{2}-1\right)}}, k_{1,3}>0$ \\
\hline$\mu_{2}, \mu_{3}$ & $\theta_{2,3}=\sqrt{\frac{K_{1}}{\left(k_{2,3}^{2}+k_{2,3}\right)} \Omega_{0}, \theta_{2,3}>0}$ & $\left(-\frac{K_{2}^{2}}{K_{1}}+4\right)+\sqrt{\left(\frac{K_{2}^{2}}{K_{1}}\right){ }^{2}+8 \frac{K_{2}^{2}}{K_{1}}}, k_{2,3}>0$ \\
\hline$\mu_{1}, \mu_{-1}\left(\frac{K_{2}^{2}}{K_{1}}-1\right)$ & $\frac{1}{1-K_{-1}}, k_{1,-1}>1$ \\
\hline$\mu_{1}, \mu_{-2}$ & $\theta_{1,-1}=\left(1-K_{-1}\right) \Omega_{0}, \theta_{1,-1}>0$ & $k_{1,-1}=\frac{1}{3-\sqrt{9+8\left(K_{-1} K_{-2}-1\right)}}, k_{1,-2}>2$ \\
\hline$\mu_{-1}, \mu_{-2}$ & $\theta_{1,-2}=\frac{\left(3-\sqrt{9+8\left(K_{-1} K_{-2}-1\right)}\right) \Omega_{0}}{4}, \theta_{1,-2}>0$ & $k_{1,-2}=\frac{K_{-1} \Omega_{0}}{3-1, k_{-1,-2}>2}$ \\
\hline
\end{tabular}
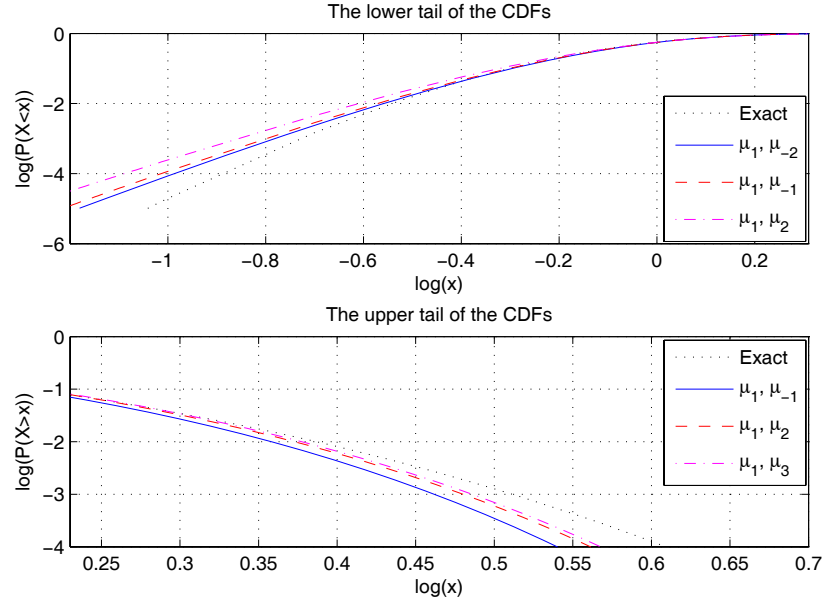

Fig. 3. The log-log CDF plots of the generalized- $K$ and the approximating Gamma RVs for $m_{m}=10$ and $m_{s}=10$ using the moment matching method.

in [12]. The value of $\mathrm{AF}_{\max }$ is determined by the smallest physical values of $m_{m}$ and $m_{s}$ which are non-zero in real propagation channels; hence $\mathrm{AF}_{\max }$ is finite.

The expressions of the scale and shape parameters given by (14a) and (14b) result in poor approximation in the lower and upper tail regions since matching only the first and second moments will result in a good fit only around the mean. To overcome this limitation, we may consider the following adjustable form for the expressions in (14a) and (14b):

$$
\begin{gathered}
\theta_{1,2}^{\prime}=[\mathrm{AF}-\epsilon] \Omega_{0}, 0 \leq \mathrm{AF} \leq \mathrm{AF}_{\max }, \epsilon_{0} \leq \epsilon \leq \mathrm{AF} \\
k_{1,2}^{\prime}=\frac{1}{\mathrm{AF}-\epsilon}, 0 \leq \mathrm{AF} \leq \mathrm{AF}_{\text {max }}, \epsilon_{0} \leq \epsilon \leq \mathrm{AF}
\end{gathered}
$$

Since the AF "added" to the scale parameter of the approximating Gamma PDF should not exceed the original amount of fading of the approximated PDF (i.e., $\epsilon_{0} \geq-\mathrm{AF}$ ), $\epsilon$ is bounded as $-\mathrm{AF} \leq \epsilon \leq \mathrm{AF}$. Due to the fact that the relevant practical range of $A F$ is from zero (for non-fading channels) to 8 (for severe multipath fading and shadowing conditions where $m_{m}=0.5$ and $\left.m_{s}=0.5\right)^{3}$, the relevant range of the adjustment factor $\epsilon$ becomes $-8 \leq \epsilon \leq 8$.

\footnotetext{
${ }^{3}$ Such small values of $m_{m}$ and $m_{s}$ may take place in land mobile satellite channels [26].
}

The adjustment factor can be computed using a numerical measure of the difference between the approximated and the approximating PDFs (CDFs). A common measure is the absolute value of the difference between the approximated and the approximating PDFs (CDFs) $[22,25]$ that is similar to the well-known Kolmogorov distance between the CDFs of two continuous distributions. For this purpose, the CDFs rather than the PDFs are considered since the Gamma PDF goes to infinity as $x \rightarrow 0$ for $k<1$ [22] which causes numerical instabilities for comparison in the lower tail region.

The plots of the optimal adjustment factor, $\epsilon_{o p}$, versus the multipath fading and shadowing parameters are shown in Figs. 4 and 5 for values of $m_{m}$ and $m_{s}$ ranging from 0.5 to 10 . The plots show that the adjustment factor decreases as either or both $m_{m}$ and $m_{s}$ increase. The decrease of the adjustment factor as both $m_{m}$ and $m_{s}$ increase is worth noting since it indicates that the PDF of the product of two Gamma RVs can be well-approximated, for the main body of the PDF, by a Gamma PDF by matching the first two positive moments. This is due to the fact that both PDFs approach the same limiting PDF (the Dirac delta PDF) as fading diminishes. To see that, the $\mathrm{AF}$ for equal values of the multipath fading and shadowing parameters can be expressed as $\mathrm{AF}=\frac{2 m+1}{m^{2}}$, where $m=m_{m}=m_{s}$; clearly the amount of fading is approximately $2 / m$ for moderate values of $m$ and converges to zero for very large values of $m$. However, if a high degree of accuracy is sought in the lower tail region, then the magnitude of the adjustment factor increases as seen in Fig. 5. Similar plots can be obtained for any region of interest and the corresponding adjustment factor can be tabulated.

\section{ON The Approximation OF The PDF OF THE Sum of INDEPENDENT GENERALIZED- $K$ RVS}

The moment matching method can be used to approximate the PDF (CDF) of the sum of $N$ generalized- $K$ RVs by a Gamma PDF (CDF). However, matching the higher order moments is difficult since deriving or computing these moments is involved or unfeasible [23, 24]. This motivates again the use of an adjustable form for the scale and shape parameters obtained by matching the first two positive moments.

We may start with $N=2$ where the first and second moments of the sum of two independent RVs, $z=x+y$, can be 


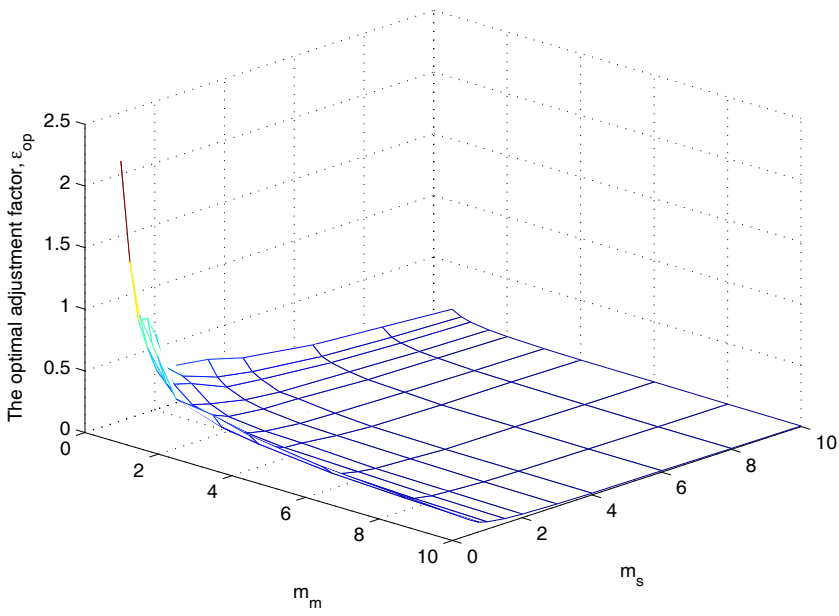

Fig. 4. The plot of the adjustment factor that minimizes the absolute value of the difference between the approximated generalized- $K$ and the approximating Gamma distributions over the whole CDF.

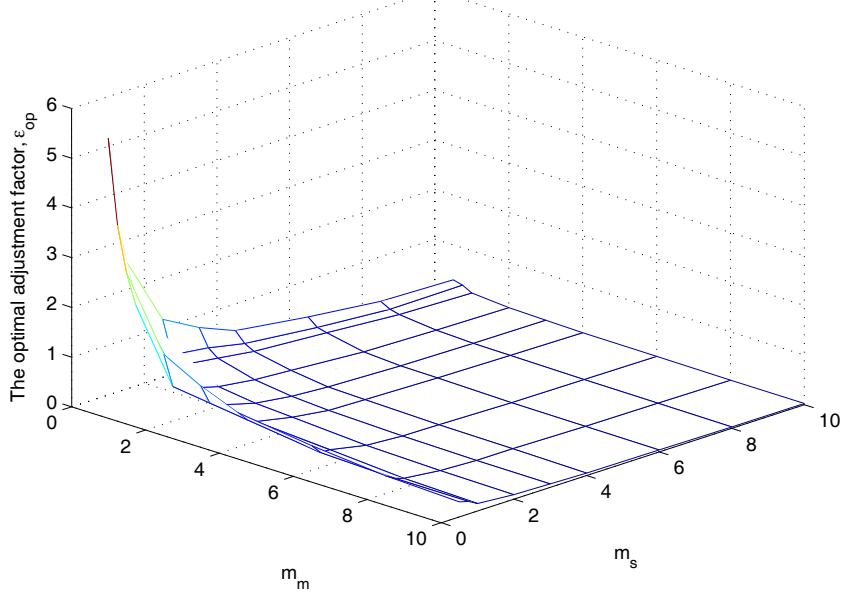

Fig. 5. The plot of the adjustment factor that minimizes the absolute value of the difference between the generalized- $K$ and the approximating Gamma distributions in the lower tail of the $\mathrm{CDF}(<0.1)$.

expressed as [22]

$$
E[z]=E[x]+E[y]
$$

and

$$
E\left[z^{2}\right]=E\left[x^{2}\right]+E\left[y^{2}\right]+2 E[x] E[y] .
$$

Matching the first and second moments of the sum of two independent generalized- $K$ RVs and the approximating Gamma distribution results in

$$
\begin{aligned}
\theta_{\text {sum }}= & \frac{K_{1, x} \Omega_{0, x}^{2}+K_{1, y} \Omega_{0, y}^{2}+2\left(\Omega_{0, x} \Omega_{0, y}\right)-\left(\Omega_{0, x}+\Omega_{0, y}\right)^{2}}{\left(\Omega_{0, x}+\Omega_{0, y}\right)} \\
= & \frac{\mathrm{AF}_{x} \Omega_{0, x}^{2}+\mathrm{AF}_{y} \Omega_{0, y}^{2}}{\left(\Omega_{0, x}+\Omega_{0, y}\right)}, \theta_{\text {sum }}>0,
\end{aligned}
$$

and

$$
k_{\text {sum }}=\frac{\left(\Omega_{0, x}+\Omega_{0, y}\right)^{2}}{\mathrm{AF}_{x} \Omega_{0, x}^{2}+\mathrm{AF}_{y} \Omega_{0, y}^{2}}, k_{\text {sum }}>0,
$$

where $K_{1, x}$ and $K_{1, y}$ denote the $K_{1}$ parameters (as defined in (13-a)), $\Omega_{0, x}$ and $\Omega_{0, y}$ denote the values of the mean of

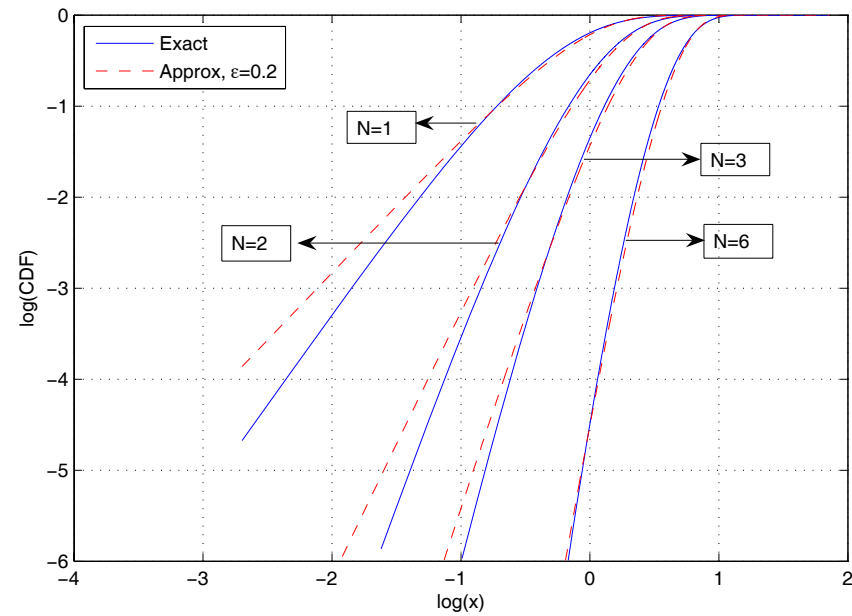

Fig. 6. The log-log CDF plots for the sum of generalized- $K$ RVs and the approximating Gamma RV for $m_{m}=2, m_{s}=4\left(\sigma_{s}=4.2 \mathrm{~dB}\right), \epsilon=0.2$, and different values of $N$.

the local power, and $\mathrm{AF}_{x}$ and $\mathrm{AF}_{y}$ denote the $\mathrm{AF}$ (as given in (14a)) of the generalized- $K$ RVs $x$ and $y$, respectively.

The adjusted forms of (17a) and (17b) can be written as

$$
\theta_{\text {sum }}^{\prime}=\frac{\left[\mathrm{AF}_{x}-\epsilon_{x}\right] \Omega_{0, x}^{2}+\left[\mathrm{AF}_{y}-\epsilon_{y}\right] \Omega_{0, y}^{2}}{\left(\Omega_{0, x}+\Omega_{0, y}\right)}, \theta_{\text {sum }}^{\prime}>0,
$$

and

$$
k_{\text {sum }}^{\prime}=\frac{\left(\Omega_{0, x}+\Omega_{0, y}\right)^{2}}{\left[\mathrm{AF}_{x}-\epsilon_{x}\right] \Omega_{0, x}^{2}+\left[\mathrm{AF}_{y}-\epsilon_{y}\right] \Omega_{0, y}^{2}}, k_{\text {sum }}^{\prime}>0 .
$$

In general, the expressions in (18a) and (18b) can be generalized for the sum of $N$ independent generalized- $K$ RVs as

$$
\theta_{\text {sum }}^{\prime}=\frac{\sum_{i=1}^{N}\left[\mathrm{AF}_{i}-\epsilon_{i}\right] \Omega_{0, i}^{2}}{\sum_{i=1}^{N} \Omega_{0, i}}, \theta_{\text {sum }}^{\prime}>0,
$$

and

$$
k_{\text {sum }}^{\prime}=\frac{\left(\sum_{i=1}^{N} \Omega_{0, i}\right)^{2}}{\sum_{i=1}^{N}\left[\mathrm{AF}_{i}-\epsilon_{i}\right] \Omega_{0, i}^{2}}, k_{\text {sum }}^{\prime}>0 .
$$

For the i.i.d. case, the expressions in (19a) and (19b) simplify to

$$
\theta_{\text {sum }}^{\prime}=(\mathrm{AF}-\epsilon) \Omega_{0}, \theta_{\text {sum }}^{\prime}>0,
$$

and

$$
k_{\text {sum }}^{\prime}=\frac{N}{\mathrm{AF}-\epsilon}, k_{\text {sum }}^{\prime}>0 .
$$

Similar formulation can be carried out for the sum of correlated generalized- $K$ RVs.

Three-dimensional plots of the adjustment factor versus the composite fading parameters $m_{m}$ and $m_{s}$ can be produced for different values of $N$. As an example, the plots of the lower tail of the CDFs for $m_{m}=2, m_{s}=4$, and $N=1,2,3$, and 6 are given in Fig. 6 showing that an adjustment factor of $\epsilon=0.2$ results in almost identical CDFs, in the lower tail region, for $N=6$. Clearly, larger values of $\epsilon$ are needed for a more accurate approximation for $N=1,2$, and 3 . 
Remark: Another approach to approximate the PDF of the sum of independent generalized- $K$ RVs can be based on the fact that the lower and upper tails of the PDF of the sum of independent positive RVs are due to the convolution of the lower and upper tails of the corresponding individual PDFs, respectively. So, the results obtained in Section IV can be used to approximate the PDF of the sum of $N$ i.i.d. generalized$K$ RVs by the PDF of the sum of the approximating $N$ i.i.d. Gamma RVs. It is well-known that the sum of $N$ i.i.d. Gamma $\mathrm{RVs}$, with the same shape and scale parameters $k_{1,2}^{\prime}$ and $\theta_{1,2}^{\prime}$, respectively, is another Gamma RV whose shape and scale parameters are $N k_{1,2}^{\prime}$ and $\theta_{1,2}^{\prime}$, respectively; these are the same as the ones obtained in (20a) and (20b). For the nonidentically distributed case, the existing results in literature on the distribution of the sum of independent non-identically distributed Gamma RVs can be utilized [27-28].

\section{Applications}

The introduced region-wise approximation for the generalized- $K$ distribution using the familiar Gamma distribution can be utilized in the performance analysis of different communication schemes over composite fading channels. So, using the closed-form expressions for the different performance metrics that are already developed for Nakagami fading channels, we present in this section examples on the use of the proposed simplifying approximation to compute some of these metrics.

\section{A. Outage Probability}

The outage probability corresponds to the probability that the received signal power falls bellow a specific threshold $\gamma_{t h}$ and can be expressed as

$$
P_{\text {out }}\left(\gamma_{t h}\right)=\operatorname{Pr}\left\{\gamma \leq \gamma_{t h}\right\}=\int_{0}^{\gamma_{t h}} p_{\gamma}(x) d x .
$$

In [29], an expression of the outage probability, for $N=1$, alternative to the one given in [13] was developed; however, the result in [29] is valid only for integer values of $m_{m}$ whereas the approximation introduced here applies for both integer and non-integer values of $m_{m}$ and $m_{s}$ including the case $m_{m}<1$. So, the outage probability can be computed by the simple CDF of the approximating Gamma distribution as

$$
P_{\text {out }}\left(\gamma_{t h}\right)=\frac{\gamma\left(k, \frac{\gamma_{t h}}{\theta}\right)}{\Gamma(k)},
$$

where $\gamma(k, x)$ is the incomplete Gamma function defined as $\gamma(k, x)=\int_{0}^{x} t^{k-1} e^{-t} d t$ [20, eq. 8.350.1].

\section{B. Outage Capacity of SIMO Channels}

The outage capacity for single-input multiple-output (SIMO) channels is determined by the probability that the instantaneous mutual information does not exceed a target rate $R$ [30]:

$$
P_{\text {out }}(R)=\operatorname{Pr}\left(\log _{2}\left[1+\mathrm{SNR} \sum_{i=1}^{N} \gamma_{i}\right] \leq R\right),
$$

where $\gamma_{i}$ denotes the instantaneous power at the $i^{t h}$ receive antenna (out of $N$ antennas) and SNR is the signal-to-noise ratio defined at the input. Using the result in Section $\mathrm{V}$ on the PDF of the sum of $N$ independent generalized- $K \mathrm{RVs}$, the outage capacity can be computed for different values of $m_{m}$ and $m_{s}$, and for different SNRs. Re-writing the expression in (23) as

$$
P_{\text {out }}(R)=P\left(\sum_{i=1}^{N} \gamma_{i} \leq \frac{2^{R}-1}{\mathrm{SNR}}\right)=\int_{0}^{\frac{2^{R}-1}{\mathrm{SNR}}} p_{\Sigma_{i} \gamma_{i}}(x) d x,
$$

the outage capacity can be computed using the familiar CDF of the Gamma RV that approximates the CDF of the sum of the $N$ independent generalized- $K$ RVs.

\section{Bit Error Rate (BER)}

Another common measure in performance analysis is the bit error rate (BER) which can be expressed as

$$
P_{e}=\int_{0}^{\infty} P_{e}(x) p_{\gamma}(x) d x,
$$

where $P_{e}(x)$ denotes the BER in an Additive White Gaussian Noise (AWGN) channel. The BER for different modulation schemes can be computed using the approximating Gamma PDF with the appropriate adjustment factor for each SNR value. So, the adjustment factor has to vary with the operating SNR for the best match. The BER for differential phase shift keying (DPSK) signaling is shown in Fig. 7 (see [12]). The values of the adjustment factor used, at $S N R=0,5,10$, $15,20,25,30 \mathrm{~dB}$ are $\epsilon=0.1,0.21,0.26,0.31,0.33,0.34$, 0.36 (for $m_{m}=1$ and $m_{s}=5$ ), $\epsilon=0.15,0.26,0.35,0.42,0.50$, $0.53,0.56$ (for $m_{m}=2$ and $m_{s}=2$ ), and $\epsilon=0.70,0.95,1.10$, $1.32,1.42,1.5,1.57$ (for $m_{m}=0.8$ and $m_{s}=1.2$ ). The values of the appropriate $\epsilon$, in all cases, were found to lie in the range $\epsilon_{o p, 1} \leq \epsilon \leq \epsilon_{o p, 0.01}$ where $\epsilon_{o p, 1}$ and $\epsilon_{o p, 0.01}$ denote the optimal adjustment parameters corresponding to the whole $\mathrm{CDF}$ and to the lower portion of the CDF $(<0.01)$, respectively. These results show that (i) the BER will depend more and more on the lower tail of the PDF as the SNR increases [15], (ii) the region-wise approximation is more needed for cases where the fading and shadowing parameters are small and close to each other. In general, analytical expressions of the BER for different transmission/reception schemes, using the approximate Gamma model, are the same as the ones obtained for Nakagami fading channels in [14].

\section{Ergodic Capacity of SIMO Channels}

The ergodic capacity of a SIMO channel can be expressed as [30]

$$
\begin{aligned}
C_{\text {erg }} & =E_{\|\mathbf{h}\|^{2}}\left[\log _{2}\left(1+\mathrm{SNR}\|\mathbf{h}\|^{2}\right)\right] \\
& =\int_{0}^{\infty} \log _{2}(1+\operatorname{SNR} x) p_{\|\mathbf{h}\|^{2}}(x) d x,
\end{aligned}
$$

where $\|\cdot\|$ denotes the norm of the single channel vector $\mathbf{h}$.

Now, since the PDF of the sum of $N$ independent generalized- $K$ RVs is approximated by a Gamma PDF, we may write

$$
C_{\text {erg }}=\frac{1}{\Gamma(k) \theta^{k}} \int_{0}^{\infty} \log _{2}(1+\operatorname{SNR} x) x^{k-1} \exp \left(-\frac{x}{\theta}\right) d x .
$$

Now using the obtained expression of the ergodic capacity for Nakagami fading channels in [14], the ergodic capacity of a 


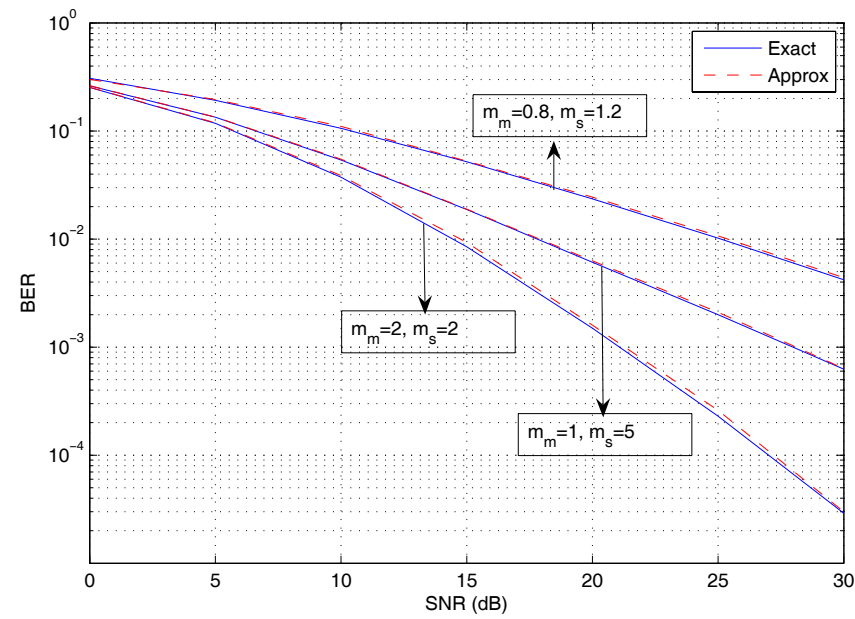

Fig. 7. The BER for DPSK signaling calculated using the proposed approximation.

SIMO system over a generalized- $K$ composite fading channel can be closely approximated, for integer values of $k$, as

$$
C_{\text {erg }}=\log _{2}(e) \exp (\eta) \sum_{j=0}^{k-1} \frac{\Gamma(-j, \eta)}{\eta^{-j}}, \eta>0
$$

where $\eta=\frac{k_{\text {sum }}^{\prime}}{\text { SNR }}$ and $\Gamma(a, x)$ is the complementary incomplete Gamma function as defined in [20, eq. 8.350.2].

Similar to the BER measure, the adjustment factor that results in the best approximation of the ergodic capacity is dependent on the operating SNR. However, the ergodic capacity is not as sensitive as the BER to numerical inaccuracy. The ergodic capacity of a heavily shadowed Rayleigh channel $\left(m_{s}=1\right)$ is shown in Fig. 8 where the loss in capacity, at high SNR, due to heavy shadowing is $1.66 \mathrm{bits} / \mathrm{s} / \mathrm{Hz}$ as compared to $0.83 \mathrm{bits} / \mathrm{s} / \mathrm{Hz}$ for Rayleigh channels without shadowing [30]. In Fig. 8, the value of the adjustment factor (for $m_{s}=1$ ) is chosen, for all SNRs, to be the average of $\epsilon_{o p, 1}$ and $\epsilon_{o p, 0.1}$ (corresponding to the lower one-tenth portion of the $\mathrm{CDF}$ ); i.e., $\epsilon=\left(\epsilon_{o p, 1}+\epsilon_{o p, 0.1}\right) / 2$. For more severe shadowing conditions with $m_{s}=0.5\left(\sigma_{s}=9 \mathrm{~dB}\right)$, the loss increases to $2.6 \mathrm{bits} / \mathrm{s} / \mathrm{Hz}$ and is well-predicted by the approximating Gamma PDF using $\epsilon=2.1$.

In Fig. 9, the ergodic capacity of the generalized- $K$ channel model and the approximating Gamma PDF for $m_{m}=2$ and $m_{s}=1.0931$ (as in [29]) is shown. Again the value of the adjustment factor, for $N=1$, is chosen in the same way as in Fig. 8 showing that a sufficient accuracy for the ergodic capacity, as compared to the BER, can be obtained through the use of a single average value of $\epsilon$. It can be seen that the use of the unadjusted values of the scale and shape parameters results in a very good match for $N=4$ since a small adjustment factor is needed. The obtained ergodic capacity for $N=4$ is different from the one in [29] since the latter does not correspond to the sum of $N$ i.i.d. generalized- $K \mathrm{RVs}$; it rather corresponds to the case when the multipath components are i.i.d. but the shadowing components are identically distributed and fully correlated.

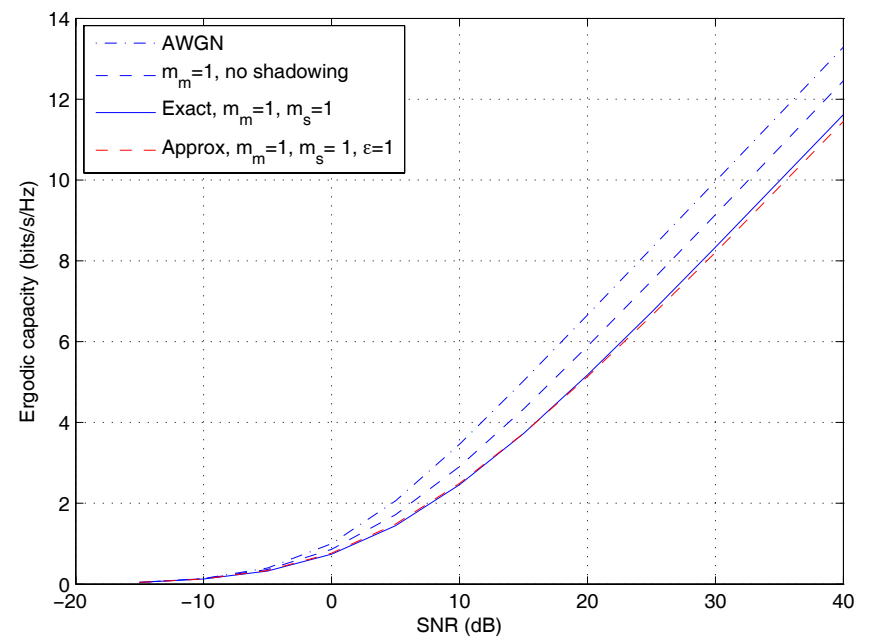

Fig. 8. The ergodic capacity plot of a Rayleigh fading channel with and without shadowing.

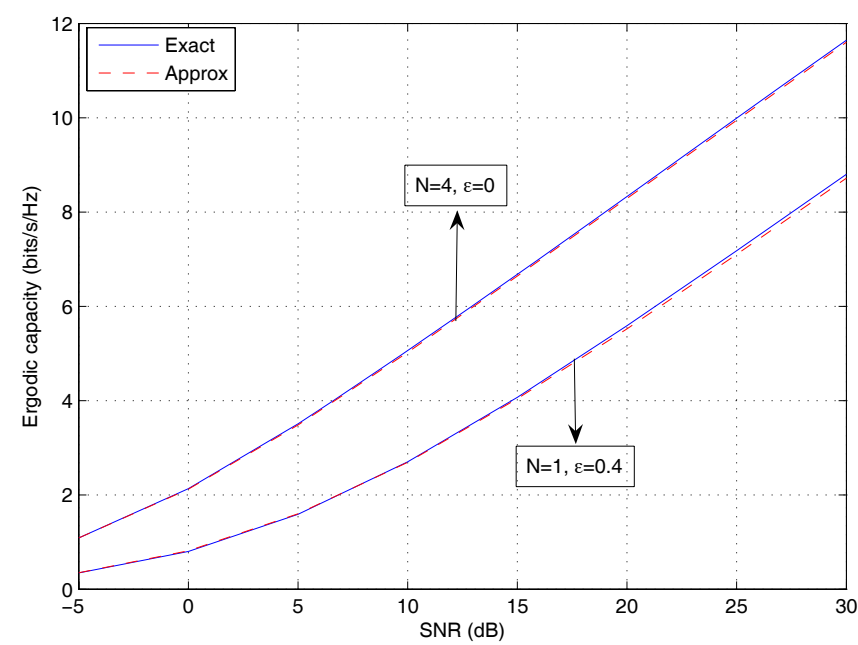

Fig. 9. The ergodic capacity plot of a shadowed Nakagami channel with $m_{m}=2$ and $m_{s}=1.0931$ for $N=1$ and $N=4$.

\section{CONCLUSIONS}

In this paper, we propose to approximate the generalized$K$ distribution by the familiar Gamma distribution through the use of the moment matching method. To avoid involved expressions when matching the higher order moments and limiting cases for small multipath fading and shadowing parameters, an adjusted form of the expressions of the parameters of the approximating Gamma distribution obtained by matching the first two positive moments is proposed. The obtained results show that the introduced adjustment results in Gamma PDFs that closely approximate the generalized$K$ distribution in both the lower and upper tail regions and can be further used to approximate the distribution of the sum of independent generalized- $K$ RVs in these regions. This sufficiently accurate region-wise approximation using the tractable Gamma distribution can significantly simplify the performance analysis of composite fading channels using measures such as probability of outage, outage capacity, and ergodic capacity. 


\section{ACKNOWLEDGMENT}

The authors would like thank Sebastian Szyszkowicz for his helpful discussions during early stages of this work. We would like also to thank the anonymous reviewers for their suggestions and comments.

\section{REFERENCES}

[1] A. J. Coulson, A. G. Williamson, and R. G. Vaughan, "A statistical basis for lognormal shadowing effects in multipath fading channels," IEEE Trans. Commun., vol. 46, no. 4, pp. 494-502, Apr. 1998.

[2] H. Suzuki, "A statistical model for urban multipath propagation," IEEE Trans. Commun., vol. COM-25, no. 7, pp. 673-680, July 1977.

[3] G. L. Stuber, Principles of Mobile Communication, 2nd ed. Boston, MA: Kluwer, 2001.

[4] A. Abdi and M. Kaveh, " $K$-distribution: an appropriate substitute for Rayleigh-lognormal distribution in fading-shadowing wireless channels," Electron. Lett., vol. 34, no. 9, pp. 851-852, Apr. 1998.

[5] A. Abdi and M. Kaveh, "On the utility of Gamma PDF in modeling shadow fading (slow fading)," in Proc. IEEE Veh. Technol. Conf., vol. 3, pp. 2308-2312, May 1999.

[6] J. Salo, L. Vuokko, H. M. El-Sallabi, and P. Vainikainen, "An additive model as a physical basis for shadow fading," IEEE Trans. Veh. Technol., vol. 56, no. 1, pp. 13-26, Jan. 2007.

[7] P. M. Kostic, "Analytical approach to performance analysis for channel subject to shadowing and fading," IEE Proc. Commun., vol. 152, no. 6, pp. 821-827, Dec. 2005.

[8] D. J. Lewinsky, "Nonstationary probabilistic target and clutter scattering models," IEEE Trans. Antenna Propag., vol. AP-31, no. 3, pp. 490-498, May 1983.

[9] M. Gu and D. A. Abraham, "Using McDaniel's model to represent non-Rayleigh reverberation," IEEE Trans. Oceanic Eng., vol. 26, pp. 348-357, July 2001.

[10] P. M. Shankar, "Error rates in generalized shadowed fading channels," Wireless Personal Commun., vol. 28, no. 4, pp. 233-238, Feb. 2004.

[11] P. M. Shankar, "Outage probabilities in shadowed fading channels," IEE Proc. Commun., vol. 152, no. 6, pp. 828-832, Dec. 2005.

[12] P. M. Shankar, "Performance analysis of diversity combining algorithms in shadowed fading channels," Wireless Personal Commun., vol. 37, no. 1-2, pp. 61-72, Apr. 2006.

[13] P. S. Bithas, N. C. Sagias, P. T. Mathiopoulos, G. K. Karagiannidis, and A. A. Rontogiannis, "On the performance analysis of digital communications over generalized- $K$ fading channels," IEEE Commun. Lett., vol. 5, no. 10, pp. 353-355, May 2006.

[14] M. K. Simon and M.-S. Alouini, Digital Communication over Fading Channels, 2nd ed. New York: Wiley, 2005.

[15] Z. Wang and G. B. Giannakis, "A simple and general parameterization quantifying performance in fading channels," IEEE Trans. Commun., vol. 51, no. 8, pp. 1389-1398, Aug. 2003.

[16] M. Nakagami, "The $m$-distribution: a general formula of intensity distribution of rapid fading," Statistical Methods in Radio Propagation, W. G. Hoffman, Ed. Oxford, UK: Pergamon Press, 1960.

[17] M. D. Springer and W. E. Thompson, "The distribution of products of Beta, Gamma and Gaussian random variables," SIAM J. Applied Maths., vol. 18, pp. 721-737, 1970.

[18] M. D. Springer, The Algebra of Random Variables. New York: Wiley, 1979.

[19] R. Barakat, "Weak-scatterer generalization of the $K$-density function with application to laser scattering in atmospheric turbulence," J. Opt. Soc. Am. A., vol. 3, no. 4, pp. 401-409, Apr. 1986.

[20] I. S. Gradshteyn and I. M. Ryzhik, Table of Integrals, Series and Products, 6th ed. San Diego, CA: Academic Press, 2000.

[21] P. S. Bithas, P. T. Mathiopoulos, and S. A. Kotsopoulos, "Diversity reception over generalized- $\left(K_{G}\right)$ fading channels," IEEE Trans. Wireless Commun., vol. 6, no. 12, pp. 4238-4243, Dec. 2007.

[22] A. Papoulis and S. U. Pillai, Probability, Random Variables, and Stochastic Processes, 4th ed. New York: McGraw-Hill, 2001.

[23] N. Cressie, A. S. Davis, J. L. Folks, and G.E. Policello, "The momentgenerating function and negative integer moments," American Statistician, vol. 35, no. 3, pp. 148-150, Aug. 1981.
[24] J. C. S. Santos Filho, P. Cardieri, and M. D. Yacoub, "Highly accurate range-adaptive lognormal approximation to lognormal sum distributions," Electron Lett., vol. 42, no. 6, Mar. 2006.

[25] N. C. Beaulieu and Q. Xie, "An optimal lognormal approximation to lognormal sum distributions," IEEE Veh. Technol., vol. 53, pp. 479-489, Mar. 2004.

[26] A. Abdi, W. C. Lau, M.-S. Alouini, and M. Kaveh, "A new simple model for land mobile satellite channels: first- and second-order statistics," IEEE Trans. Wireless Commun., vol. 2, no. 3, pp. 519-528, May 2003.

[27] P. G. Moschopoulos, "The distribution of the sum of independent gamma random variables," Ann. Inst. Statist. Math. (Part A), vol. 37, pp. 541544, 1985.

[28] G. K. Karagiannidis, N. C. Sagias, and T. A. Tsiftsis, "Closed-form statistics for the sum of squared Nakagami- $m$ variates and its applications," IEEE Commun. Lett., vol. 54, no. 8, pp. 1353-1359, Aug. 2006.

[29] A. Laourine, M.-S. Alouini, S. Affes, and A. Stéphenne, "On the capacity of generalized- $K$ fading channels," IEEE Trans. Wireless Commun., vol. 7, no. 7, pp. 2441-2445, July 2008.

[30] D. N. C. Tse and P. Viswanath, Fundamentals of Wireless Communications. Cambridge University Press, 2005.

Saad Al-Ahmadi received his M.Sc. degree in electrical engineering from King Fahd University of Petroleum \& Minerals (KFUPM), Dhahran, Saudi Arabia in April 2002 and joined the the Department of Electrical Engineering at KFUPM as a lecturer in August 2003. He is currently pursuing his Ph.D degree in electrical engineering at Carleton University, Ottawa, Canada. His research areas are statistical modeling of wireless channels and coordinated multi-point transmission and reception schemes in future cellular systems.

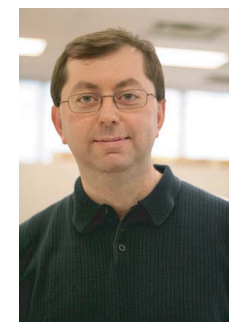

Halim Yanikomeroglu received a B.Sc. degree in Electrical and Electronics Engineering from the Middle East Technical University, Ankara, Turkey, in 1990, and a M.A.Sc. degree in Electrical Engineering (now ECE) and a Ph.D. degree in Electrical and Computer Engineering from the University of Toronto, Canada, in 1992 and 1998, respectively. He was with the R\&D Group of Marconi Kominikasyon A.S., Ankara, Turkey, from 1993 to 1994.

Since 1998 Dr. Yanikomeroglu has been with the Department of Systems and Computer Engineering at Carleton University, Ottawa, where he is now an Associate Professor. Dr. Yanikomeroglu's research interests cover many aspects of the physical, medium access, and networking layers of wireless communications. Dr. Yanikomeroglus's research is currently funded by Samsung (SAIT, Korea), Huawei (China), Communications Research Centre Canada (CRC), and NSERC. Dr. Yanikomeroglu is a recipient of the Carleton University Research Achievement Award 2009.

Dr. Yanikomeroglu has been involved in the steering committees and technical program committees of numerous international conferences; he has also given 17 tutorials in such conferences. Dr. Yanikomeroglu is a member of the Steering Committee of the IEEE Wireless Communications and Networking Conference (WCNC), and has been involved in the organization of this conference over the years, including serving as the Technical Program Co-Chair of WCNC 2004 and the Technical Program Chair of WCNC 2008. Dr. Yanikomeroglu is the General Co-Chair of the IEEE Vehicular Technology Conference to be held in Ottawa in September 2010 (VTC2010-Fall). Dr. Yanikomeroglu was an editor for IEEE TRANSACTIONS ON WIRELESS COMMUNicATIONS [2002-2005] and IEEE COMMUNiCATIONS SURVEYS \& TUTORIALS [2002-2003], and a guest editor for WILEY JOURNAL ON Wireless Communications \& Mobile Computing. He was an Officer of IEEE's Technical Committee on Personal Communications (Chair: 200506, Vice-Chair: 2003-04, Secretary: 2001-02), and he was also a member of the IEEE Communications Society's Technical Activities Council (2005-06).

Dr. Yanikomeroglu is also an adjunct professor at King Saud University, Riyadh, Saudi Arabia; he is a member of the Carleton University Senate, and he is a registered Professional Engineer in the province of Ontario, Canada. 